\title{
A REMARK ON BOUNDED STRICTLY PLURISUBHARMONIC EXHAUSTION FUNCTIONS
}

\author{
R. MICHAEL RANGE ${ }^{\prime}$
}

\begin{abstract}
A more general version of the theorem of Diederich and Fornaess on the existence of bounded strictly plurisubharmonic exhaustion functions is proved by completely elementary methods.
\end{abstract}

The following result is well known.

THEOREM 1 (DIEDERICH-FornAESS [1]). Let $\Omega \subset \subset X$ be a pseudoconvex domain with $C^{2}$ boundary in a Stein manifold $X$. Then there are a $C^{2}$ defining function $\varphi$ for $\Omega$ on a neighborhood of $\bar{\Omega}$, and $0<\eta_{0}<1$, such that for any $0<\eta<\eta_{0}$ the function $\rho=-(-\varphi)^{\eta}$ is strictly plurisubharmonic on $\Omega$.

According to [1], near $b \Omega \varphi$ is of the form $\varphi=\sigma \cdot e^{-K \psi}$, where $\psi$ is any $C^{\infty}$ strictly plurisubharmonic function on $X, K>0$ is a (large) constant, and $\sigma$ is a suitable defining function for $\Omega$ which satisfies a special estimate; in [1], $\sigma$ is obtained from the Euclidean distance function after embedding $X$ in some $\mathbf{C}^{n}$.

In this short paper we present a somewhat more general version and a simpler proof of Theorem 1 in case $b \Omega$ is just slightly smoother, namely of class $C^{3}$. The proof does not need the embedding theorem and is completely elementary. The main point is that the special function $\sigma$ in [1] can be replaced by any $C^{3}$ defining function $r$ for $\Omega$. This may be of interest in cases where one deals with an a priori given specific $r$, or when one may want to work with an $r$ in some "normal" form.

The germ of the proof given below is contained in an earlier result of J. J. Kohn; he showed that for any $C^{\infty}$ defining function $r$ the function $\mu=-\log (-r)+K \psi$ is strictly plurisubharmonic on $\Omega \cap W$ for some neighborhood $W$ of $b \Omega$, provided $K$ is sufficiently large [2, Proof of Theorem 3.7]. Since on $\Omega \cap W-e^{-\mu}=r e^{-K \psi}=$ $\varphi$, one sees that $\varphi$ is a defining function for $\Omega$, whose level sets $\{z \in \Omega: \varphi(z)=-\varepsilon\}$ are strictly pseudoconvex for $0<\varepsilon \leqslant \varepsilon_{0}$ and $\varepsilon_{0}>0$ sufficiently small. So one is almost done! In fact, it now appears plausible that by composing $\varphi$ with a function $\chi$ on $(-\infty, 0]$ which is bounded, increasing and sufficiently convex near 0 (i.e. $\chi^{\prime \prime}(t) \nearrow \infty$ as $\left.t \rightarrow 0\right)$, for example $\chi(t)=-(-t)^{\eta}, 0<\eta<1$, the Levi form of $\rho=\chi \circ \varphi$ might become positive definite in all complex directions. Indeed, one has the following result.

Received by the editors November 26, 1979.

1980 Mathematics Subject Classification. Primary 32F15.

Key words and phrases. Pseudoconvex domain, Levi form, bounded strictly plurisubharmonic exhaustion function.

${ }^{1}$ Partially supported by NSF grant MCS78-01487. 
THEOREM 2. Let $X$ be a complex manifold and let $\Omega \subset \subset X$ be a pseudoconvex domain with $C^{3}$ boundary $b \Omega$, such that there exists a $C^{\infty}$ strictly plurisubharmonic function $\psi$ defined on a neighborhood of $b \Omega$. Let $r$ be any $C^{3}$ defining function for $\Omega$. Then there are a neighborhood $W$ of $b \Omega$ and constants $K>0,0<\eta_{0}<1$, such that for $0<\eta<\eta_{0}$ the function $\rho=-\left(-r e^{-K \psi}\right)^{\eta}$ is strictly plurisubharmonic on $\Omega \cap W$. If there exists $\psi$ strictly plurisubharmonic on $\bar{\Omega}$, then $\rho$ is strictly plurisubharmonic on $\Omega$.

First, we briefly recall some notation. We fix a Hermitian metric $\mu$ on a neighborhood of $\bar{\Omega}$ and denote the induced norm on $T_{p}^{10} X, p \in \bar{\Omega}$, by $|\cdot|_{p}$. The defining function $r$ for $\Omega$ induces the orthogonal decomposition $T_{p}^{10}=T_{p}^{\prime} \oplus T_{p}^{\prime \prime}$, where $T_{p}^{\prime}=\left\{t \in T_{p}^{10}:\left\langle\partial r_{p}, t\right\rangle=0\right\}$. Correspondingly, $t \in T_{p}^{10}$ decomposes into $t=t^{\prime}+t^{\prime \prime}$. The Levi form of a $C^{2}$ function $\varphi$ at $p \in \bar{\Omega}$ is denoted by $L_{\varphi}(p ; t, \hat{t})$, where $t, \hat{t} \in T_{p}^{1,0} ; L_{\varphi}(p ; t, t)$ is also written $L_{\varphi}(p ; t)$ or simply $L(t)$.

Proof of TheOrem 2. $\Omega$ pseudoconvex means, by definition, that

$$
L_{r}(p ; t) \geqslant 0 \text { for } p \in b \Omega \text { and } t \in T_{p}^{\prime} .
$$

The main point is to have good control of $L_{r}(p ; t)$ for $p \in \Omega$ near $b \Omega$ and arbitrary $t$. Choose a product neighborhood $W=b \Omega \times(-\varepsilon, \varepsilon)$ of $b \Omega$ and let $\pi: W \rightarrow b \Omega$ be the projection. By introducing local coordinates on a neighborhood $U \subset W$ of $q \in b \Omega$, each $T_{p}^{10}, p \in U$, is canonically identified with $\mathbf{C}^{n}$. Thus, for fixed $a \in \mathbf{C}^{n}$ there is associated $a_{p}=a_{p}^{\prime} \oplus a_{p}^{\prime \prime} \in T_{p}^{\prime} \oplus T_{p}^{\prime \prime}$, and the function $L_{r}\left(p ; a_{p}^{\prime}\right)$ is of class $C^{1}$ in $p \in U$ (this is where one needs $b \Omega$, and hence $r$ of class $C^{3}$ !). Therefore, for $p \in U$,

$$
L_{r}\left(p ; a_{p}^{\prime}\right)-L_{r}\left(\pi(p) ; a_{\pi(p)}^{\prime}\right)=O(|r(p)|)\left|a_{p}\right|_{p}^{2},
$$

and hence, by (1) and standard compactness arguments,

$$
L_{r}\left(p, t^{\prime}\right) \geqslant O(|r(p)|)|t|_{p}^{2} \quad \text { for } p \in \Omega \cap W \text { and } t \in T_{p}^{10} .
$$

(2) was given already by Kohn [2, p. 281]. Now (2),

$$
L(t)=L\left(t^{\prime}\right)+2 \operatorname{Re} L\left(t^{\prime}, t^{\prime \prime}\right)+L\left(t^{\prime \prime}\right)=L\left(t^{\prime}\right)+O\left(|t|_{p}\left|t^{\prime \prime}\right|_{p}\right)
$$

and

$$
\left|t^{\prime \prime}\right|_{p}=O\left(\left|\left\langle\partial r_{p}, t\right\rangle\right|\right)
$$

imply, for some constant $A>0$,

$$
L_{r}(p ; t) \geqslant-A|r(p)||t|_{p}^{2}-A|t|_{p}\left|\left\langle\partial r_{p}, t\right\rangle\right| \text { for } p \in \Omega \cap W \text { and } t \in T_{p}^{10} .
$$

The rest of the proof is now just a simple modification of the estimation in [1], using (5) instead of Lemma 1 in [1]. For the convenience of the reader we include the relevant steps. First, shrink $W$, so that $\psi$ is strictly plurisubharmonic on $\bar{W}$. Next, with $\rho=-\left(-r e^{-K \psi}\right)^{\eta}$, one calculates

$$
\begin{aligned}
& L_{\rho}(p, t)=\eta(-r)^{\eta-2} e^{-\eta K \psi}\{ K r^{2}\left[L_{\psi}(t)-\eta K|\langle\partial \psi, t\rangle|^{2}\right] \\
&+(-r)\left[L_{r}(t)-2 \eta K \operatorname{Re}\langle\partial r, t\rangle \overline{\langle\partial \psi, t\rangle}\right] \\
&\left.+(1-\eta)|\langle\partial r, t\rangle|^{2}\right\}
\end{aligned}
$$


for $t \in T^{10}(\Omega \cap W)$. Let $D(t)$ be the expression in \{ \}; we must show that one can choose $\eta$ and $K$ so that $D(t)>0$ for $t \neq 0$. Choose $A_{1}>0$ so that $L_{\psi}(p ; t)>A_{1}|t|_{p}^{2}$ for $t \in T^{10}(W)$. By (5) and (6), and choosing $0<\eta<\eta_{0}(K)$,

$$
D(t) \geqslant K^{2}\left[A_{1}-A_{1} / 2\right]|t|^{2}+(-r)\left[r A|t|^{2}-A_{2}|\langle\partial r, t\rangle||t|\right]+\frac{1}{2}|\langle\partial r, t\rangle|^{2}
$$

by estimating $|r| A_{2}|\langle\partial r, t\rangle||t| \leqslant \frac{1}{4}|\langle\partial r, t\rangle|^{2}+A_{3} r^{2}|t|^{2}$ in (7), one obtains

$$
D(t) \geqslant r^{2}\left[K A_{1} / 2-A_{4}\right]|t|^{2},
$$

where all the $A$ 's are positive and independent of $K, \eta$ and $t \in T^{10}(\Omega \cap W)$. So, the desired result follows by choosing $K>2 A_{4} / A_{1}$, and then $\eta_{0}=\eta_{0}(K)$.

In case $\psi$ is strictly plurisubharmonic on $\bar{\Omega}, L_{\psi}(t) \geqslant A_{5}|t|^{2}$ for $t \in T^{10}(\bar{\Omega})$ and $A_{5}>0$; also $r^{2} \geqslant \varepsilon>0$ on the compact set $C=\Omega \backslash W$. Hence one obtains from (6), which now holds on $\Omega, D(t)>K \varepsilon^{2} A_{5}|t|^{2}-A_{6}|t|^{2}$ for $t \in T^{10}(C)$; now choose $K>\max \left(2 A_{4} / A_{1}, A_{6} / \varepsilon^{2} A_{5}\right)$. Q.E.D.

Remarks. (1) It is obvious that in case $r$ (i.e. $b \Omega$ ) is of class $C^{k}, 3<k<\infty$, the function $\rho$ is $C^{k}$ on $\Omega \cap W$ (resp. $\Omega$ ) as well.

(2) I do not know whether Theorem 2 holds in case of a $C^{2}$ boundary. Also, it seems to be unknown whether there exists a bounded strictly plurisubharmonic exhaustion function for $\Omega$ in case of a $C^{1}$ boundary (cf. [1, p. 133]).

\section{REFERENCES}

1. K. Diederich and J. E. Fornaess, Pseudoconvex domains: Bounded strictly plurisubharmonic exhaustion functions, Invent. Math. 39 (1977), 129-141.

2. J. J. Kohn, Global regularity for on weakly pseudoconvex manifolds, Trans. Amer. Math. Soc. 181 (1973), 273-292.

Department of Mathematics, State University of New York at Albany, Albany, New York 12222 